\title{
Hot-stop PCR: a simple and general assay for linear quantitation of allele ratios
}

H Uejima et al.

Nature Genet. 25, 375-376 (2000).

Two related studies were brought to our attention since the publication of our manuscript. Neither study referred in its title or abstract to methods for allele quantitation and were therefore unknown to us, but we would have cited them if we had known of them. One study describes the addition of a radiolabeled primer to the final cycle of PCR to distinguish between single base pair mutations ${ }^{1}$; the other describes the addition of radioactive dNTPs to the final cycle to distinguish mitochondrial DNA species ${ }^{2}$. Neither demonstrates the ability to quantitate varying allele ratios with precision.

1. Schrijver, I., Liu, W., Brenn, T., Furthmayr, H. \& Francke, U. Cysteine substitutions in epidermal growth factor-like domains of fibrillin-1: distinct effects on biochemical and clinical phenotypes. Am. J. Hum. Genet. 65, 1007-1020 (1999).

2. Moraes, C.T., Ricci, E., Bonilla, E., DiMauro, S. \& Schon, E.A. The mitochondrial tRNA(Leu(UUR)) mutation in mitochondrial encephalomyopathy, lactic acidosis, and strokelike episodes (MELAS): genetic, biochemical, and morphological correlations in skeletal muscle. Am. J. Hum. Genet. 50, 934-949 (1992).

\section{The insulin gene VNTR is associated with fasting insulin levels and development of juvenile obesity}

\author{
C Le Stunff et al.
}

Nature Genet. 26, 444-446 (2000).

Throughout our paper, the ' $\mathrm{T}$ ' and 'A' alleles at the $H p h 1$ site were switched; ' $\mathrm{T}$ ' should be ' $\mathrm{A}$ ' and vice versa. This does not change the reported results regarding the VNTR polymorphism. 